\title{
Application of Perceptron Networks in Recommending Medical Diagnosis
}

\author{
Venkata Karthik Gullapalli \\ Vellore Institute of Technology \\ School of Computing Science \\ and Engineering \\ VIT University, India
}

\author{
Rahul Brungi \\ Vellore Institute of Technology \\ School of Computing Science \\ and Engineering \\ VIT University, India
}

\author{
Gopichand G \\ Vellore Institute of Technology \\ School of Computing Science \\ and Engineering \\ VIT University, India
}

\begin{abstract}
Artificial intelligence applications in medicine is the major and evolutionary topic in the technology world. Neural networks is an important branch of machine learning which is inspired from biological neural networks. Neural networks are useful in making proper decisions in rational environments with uncertainty. The neural networks perform better computation with high power with the help of the multiple interconnected neurons which act as processing elements. Decision theory along with probabilistic theory gives the good way to make the right decisions. Neural Network systems help in linking the health observations with the health knowledge database to take better decisions for good health. The ability of a neural network to learn by example can be implemented for taking decisions that would increase the rate of providing the better medical care facilities. This paper presents a novel methodology to implement supervised learning networks that is perceptron networks in medical diagnosis for providing such good decisions to the doctors in helping patients by also providing a good health care.
\end{abstract}

\section{General Terms}

Neural Networks, Decision Making

\section{Keywords}

Clinical Decision Support System, Perceptron, Processing Elements, Weights, Activation Functions, healthcare system

\section{INTRODUCTION}

Neural networks are the old algorithms that are motivated by the goal of having machines that can mimic the brain. The origin of the neural networks was the algorithms that try to mimic the brain. Brain is the most amazing learning machine. The aim of the neural networks is to emulate human mind as closely as possible. It is the collection of the methodologies that aim to exploit tolerance for imprecision and uncertainty to achieve tractability, robustness and low solution cost. In the modern world, precision and certainty carry a cost. Neural network is a processing device. It can be either an algorithm or a hardware device. The concept of neural networks is inspired by the design and functioning of brain. It gives the ability to learn by example by being very flexible and powerful at the same time. The concepts of neural networks are well suited for the real time applications because of fast response and computational time. Artificial neural networks tries to replicate the functioning of brain. They are information processing systems that are constructed and implemented to model human brain. The aim of the neural networks is to develop a computational device to perform tasks at a faster rate efficiently. Neural networks contains highly interconnected processing elements that are working in union to solve a problem and provide an efficient solution. These are configured for specific applications. The processing time for neural networks is faster than the normal computations performed by human brain and the size and complexity of neural networks depends on the practical applications. Neural networks are computationally expensive algorithms. Modern neural networks are the effective state of the art technique for many applications. Neurons can be visualized for their arrangements in layers. Neural networks basically are highly interconnected processing elements and the output of each processing element is connected through weights to the other processing element or itself. The arrangement and geometry are essential for the computation in neural networks. The origin and terminating points of a neural networks are noted and the function of each processing element should be specified. Neural networks were developed as simulating neurons or network of neurons in the brain. Each layer in a neural network is formed by a processing element and combining it with the other processing elements. A layer implies a stage and input and output stages are linked. Linked interconnections lead to various network architectures. So neuron is a computational unit that gets a number of inputs, does some computation and sends the output to other neurons in the network. The architecture followed by neural networks is basically parallel architecture. In processing element in a neural network consists of input and output parts which are regarded as two major components. The activation function is applied over the net input to calculate the output of a neuron in a neural network. Mostly nonlinear functions are used to achieve the advantages of a multilayer network over a single layer network. If a linear activation function is used in a multilayer network then the output remains same as that obtained from a single layer network. So in most of the cases nonlinear activation functions are used. There are different types of activation functions such as identity function, binary step function, sigmoidal function, hyperbolic tangent function and RAMP function. Each neuron is connected to other neurons by directed communication links in a network and each communication link is associated with a weight. These weights contain information about the input signal. Learning rate is used to control the amount of weight adjustment at each step of training and it ranges between 0 and 1 . The layers between the input layer and the output layer are hidden layers which contains processing elements. Neural networks are designed to handle unambiguous and noisy data to provide tractable solution. Neural networks have high intelligence quotient with approximate reasoning.

In figure $1, \mathrm{X} 1$ and $\mathrm{X} 2$ are the input neurons that transmit signals and $\mathrm{Y}$ is the output neuron that receives signal. So $\mathrm{X} 1$, $\mathrm{X} 2$ and $\mathrm{Y}$ are considered as processing elements that are connected in such a way that the outputs of X1 and X2 are the inputs for the neuron $\mathrm{Y}$. In figure $1, \mathrm{x} 1$ and $\mathrm{x} 2$ are the activations of the input neurons $\mathrm{X} 1$ and $\mathrm{X} 2$ respectively where are $\mathrm{w} 1$ and $\mathrm{w} 2$ are the associated weights that contain 
information about the input signals for the links between the processing elements $\mathrm{X} 1, \mathrm{Y}$ and $\mathrm{X} 2, \mathrm{Y}$ respectively. The final output of the neuron $\mathrm{Y}$ is given as $\mathrm{y}$. If $\mathrm{f}$ is an activation function then the output $y$ can be calculated as $y=f\left(y_{\text {in }}\right)$ where $\mathrm{y}_{\mathrm{in}}$ is calculated as $\mathrm{y}_{\mathrm{in}}=\left(\mathrm{x} 1{ }^{*} \mathrm{w} 1\right)+(\mathrm{x} 2 * \mathrm{w} 2)$.

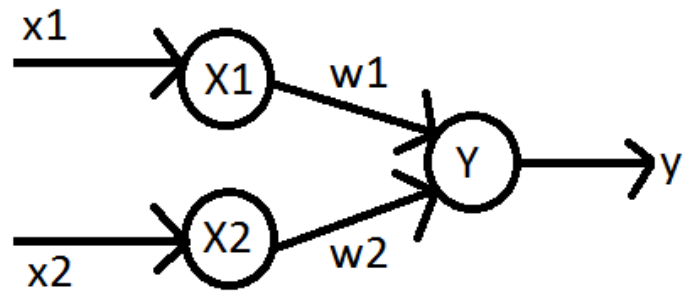

Figure 1

Artificial Neural networks store information in contiguous memory locations and some of the information may be lost due to overloading of memory. In neural networks there is a control unit in Central Processing Unit (CPU) which can transfer and control precise scalar values from unit to unit. Neural networks possess simpler interconnections between the processing elements. The main property of neural networks is learning in two ways by parameter learning and structure learning.

\section{EXISTING SYSTEM}

\subsection{Clinical Decision Support System (CDSS)}

Clinical Decision Support System is a technology that is made to help doctors and physicians in making decisions related to the health care of a patient. CDSS maps the observations with the health knowledge database to make decisions and provide the results. It uses one or more features and the symptoms of the patients to generate specific advice for the respective health problem. It uses knowledge database to extract features. The main objective of a CDSS is to achieve an advice for patients based on their health data. The purpose of the CDSS is to assist doctors. A doctor would use CDSS for diagnosing and analyzing the data of the patients. The doctor should input the patient data and wait for the CDSS to output the right decision. It requires both the knowledge of the doctor and patient to make right choices. And for providing better results. This system takes the data of patients and gives the set of diagnoses that are related to the history of the health condition of patients. The doctor then has to pick which diagnoses are relevant and then suggest the medical prescription. Doctors use this system when they are dealing with patients as it helps them make right decisions at various stages of treatment. CDSS systems are used to help the doctors in preparing the diagnoses and filter the preliminary diagnostic recommendations to improve the final results and these systems are also used to mine data to derive patterns in the past medical history of patients to predict the future events.

\subsection{Working Method}

The CDSS follows two different methods in helping doctors choose a decision. One is the knowledge base method while the other is non-knowledge based method. The knowledge base method contains the set of inference rules that can be implemented on the compiled data. These rules are made from the knowledge database based on the data of patients. CDSS that works on the non-knowledge based method uses the concepts of machine learning. This works by allowing computers learn from the past experiences and finding patterns in the health data of patients. Non-knowledge based method is usually implemented using the concepts of neural networks. Neural networks analyze the patterns in the data to derive the mappings between the symptoms and a diagnosis. In the neural networks non-knowledge based method the system cannot explain the reason for its decision so doctors do not use this system for reliability and accountability reasons. The method through neural networks focus on a narrow list of symptoms whereas knowledge based approach cover many different diseases to diagnosis. These systems focus only on the functional decision making. So when these systems gives a range of decisions, it is up to the doctors to choose the right decision. Typically the CDSS would make suggestions of outputs or a set of outputs for the doctor and the doctor picks the useful suggestions out of the set and removes the other suggestions. Though this is a good way the whole process consumes lot of time as the doctor also need to check for the better suggestions. Also the knowledge database has to be updated every now and then so that it learns from the experience in the case of neural networks and new inference rules must be implemented in the case of knowledge based methods. If there is a very small error in the data updated in the database then the CDSS would show wrong recommendations. Every detail in the hospitals must be computerized and the health information must be processed and stored in every database with accuracy and precision for using CDSS.

\subsection{Technical Challenges}

Till now CDSS achieved success but still there are a lot of challenges and barriers of implementation faced by the system. Biological systems and the data related to these systems are highly complicated and a CDSS may us potentially relevant data which is very crucial. This system potentially considers patients symptoms, medical history, family history and genetics along with the historical and geographical trends of disease occurrence respectively for different patients. It then publishes medical data when recommending a course of treatment for patients. CDSS is a standalone application which is not integrated with the healthcare systems. The extensive quantity of quality medical research should be incorporated into the CDSS very often and it should be updated frequently. Some research may be contradicting and in these cases the CDSS makes vague decisions due to the complicated and parallel theories for a certain issue. It depends on what kind of data the database is being uploaded with. Different CDSSs serves different purposes and there is no general method that is applicable to all types of CDSS. A CDSS should have consistency and accuracy in its classification of a disease. It is evaluated based on the improvement of health condition of patients. So the main challenge the CDSS faces is its accuracy and efficiency for improving the health conditions by providing right choices which depends on the data it is uploaded with. The data that is being uploaded is a very sensitive data which can be used for good purposes and equally bad purposes. So certain care and measures are to be taken while implementing CDSS and even a small decision can affect the lives of people.

\section{PROPOSED METHOD}

\subsection{Single Layer Feed Forward Network}

Neural networks are classified into single layer and multilayer neural networks along with some other different types as feed forward neural network and feed backward neural network. A layer is formed by taking a processing element and combining it with the other processing elements. The linked interconnections lead to various network architectures. In a 
single layer feed forward neural network, the input and output layers are linked with each other directly by weights and activation functions as shown in figure 2 .

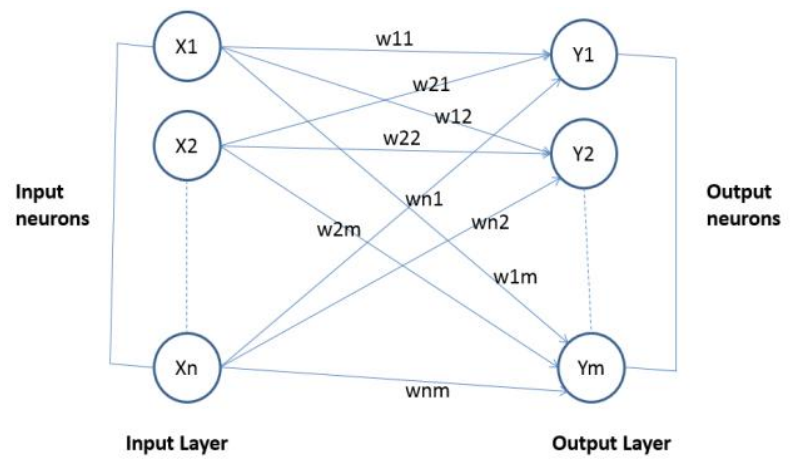

Figure 2

\subsection{Perceptron Networks}

Perceptron networks are also called as supervised learning networks and they come under the category of single layer feed forward networks. They are also called as simple perceptron networks or pattern recognizers. It consists of three units namely sensory unit, associator unit and response unit. Sensory unit acts as an input unit whereas associator unit acts as hidden unit and response unit as output unit. The sensory units are connected to associator units with fixed weights having values $+1,0,-1$ assigned randomly. The binary activation function is used in sensory unit and associator unit. The response unit has an activation of $+1,0$ or -1 . The binary step with fixed threshold theta is used as an activation for the associator. The output signals that are sent from the associator unit to the response unit are binary and the output of the perceptron network is given by $\mathrm{y}=\mathrm{f}\left(\mathrm{y}_{\text {in }}\right)$.

The activation function $f\left(y_{\text {in }}\right)$ is given the values 1 when $y_{\text {in }}$ greater than theta, 0 when $y_{\text {in }}$ lies between negative of theta and theta and -1 when $y_{\text {in }}$ less than negative of theta.

Perceptron learning rule is used in the weight updating between the associator unit and the response unit. For each training input the net will calculate the response and it will determine whether or not an error has occurred. The error calculation is based on the comparison of the values of targets with those of the calculated outputs. The weights on the connections from the units that send the nonzero signal will get adjusted. The weights will be adjusted based on the learning rule and if an error has occurred for a particular training pattern or not.

The following equations are used for upgrading the weight and the bias.

$$
\mathbf{w}_{\mathbf{i}}(\text { new })=\mathbf{w}_{\mathbf{i}}(\text { old })+\left(\text { alpha } * \mathbf{t} * \mathbf{x}_{\mathbf{i}}\right)
$$$$
b(\text { new })=b(\text { old })+(\text { alpha } * t)
$$

In the above equations, $t$ is the target value which is equal to +1 or -1 and alpha is the learning rate. If there are no errors then the training process is stopped. The learning signal is the difference between the desired and the actual response of a neuron that is the error signal. A sensory unit generates the signals for the associator unit. The weights between the sensory unit and the associator unit are fixed and cannot be changed. These weights are chosen arbitrarily from $+1,0$ and -1 . The weights between the associator unit and the response unit can only be changed. Figure 3 shows the single classification perceptron network architecture.

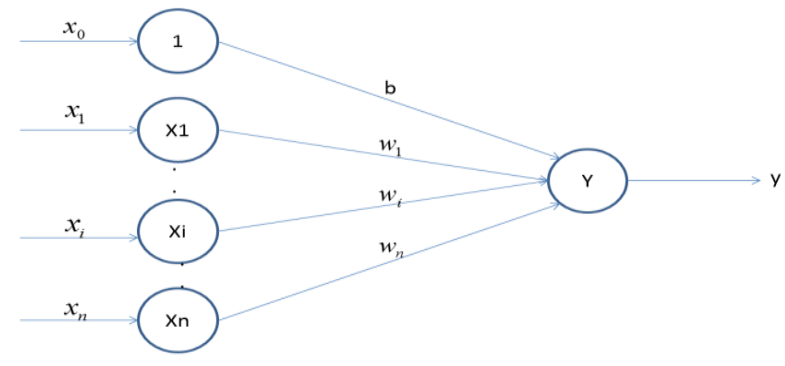

Figure 3

The network performance of a perceptron network is tested once the training process is over. To increase the efficiency of the network, it should be trained with more number of datasets. The testing of the perceptron network is done by classifying the known outputs.

\subsection{Working Method}

Neural networks are used to construct efficient computer systems that can learn from the experience and produce accurate results in the environment of uncertainty and imprecision. In the present paper a supervised learning perceptron neural network is proposed to make decisions for medical diagnosis. Neural networks offer a very powerful computation system for mapping several input variables to output variables.

Neural networks are trained with large amounts of data and rules that are feed as input to them. The medical data that is given to these neural networks are accurate and precise. For this purpose the database is first uploaded with large amounts of medical data. All this data belongs to the existing health problems and it also contains the health information, genetics and the previous records of every patients. When entering every record into the database, precision is taken as an important attribute and the database contains all the data without any flaws. The inference rules are written for the symptoms that acts as inputs for processing and providing decisions. Then the inference rules are applied on this data so that the database initially process certain information before feeding that to the neural network. This method differs from CDSS as in this method the knowledge database is combined with the perceptron networks for multiple computations with accuracy. In CDSS they are classified as knowledge based and non-knowledge based methods. The knowledge database provides the perceptron network with the proper data for further computation in the proposed method. After the knowledge base of medical records is computed, the output of the knowledge base is provided as an input to the supervised learning algorithm. In supervised learning algorithm, the data and the previous medical records are taken and analyzed to predict the outputs for the new inputs. The outputs of the knowledge base acts as the new input for the supervised learning algorithm and the algorithms predicts the possible outputs based on its previous training data and experience. The outputs predicted by the supervised learning algorithm are given as inputs to the neural network. The input features that are given in the beginning to the database are further classified and processed during the knowledge database processing and in the computation performed by the supervised learning algorithm. This process gives the efficient results to make the decisions as the knowledgebase and supervised learning algorithm acts as filters to reduce the number of decisions to make for accuracy and precision. Neural networks are used as nodes to derive the relationship between the symptoms and diagnosis. The neural networks 
then takes the input data and performs an efficient computation. The data in the knowledge database is first classified using the supervised learning rule. This classifies the data properly. The neural network is trained thoroughly where the neural network is not given proper information about the previous inputs but it is capable of extracting the relevant information from the data that is given as input to generate the clusters and to provide the doctors with the decisions that are more relevant and precisely matching the medical history and symptoms of the patients. Supervised learning refers to a fact that the algorithm is given with datasets in which the correct medical data is provided and the task of the algorithm here is to produce the right predictions based on the medical records. This method reduces the decisions to be made to a small number by considering all the relevant information and provides the decisions to the doctors that are more accurately related to the symptoms of patients and which in return reduces the number of alerts. This system does not need any input from the doctors every time as it uses the medical history of patients stored in the databases and processes the information accordingly. In this way the system can also work on the data that is not complete and accurate by using the previous records from the database and completing the data based on the precise assumptions so that the system provides the decisions with high efficiency and help doctors in making the right choice from the provided correct decisions.

\section{Medical Data and Decisions}

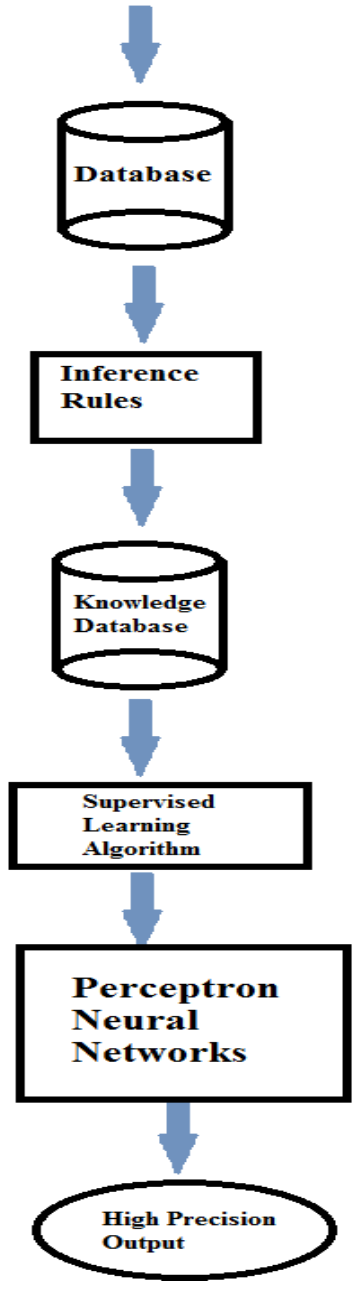

Figure 4
What basically is happening is the database with proper medical records is set up with certain inference rules and then the data is feed to supervised learning algorithm. The output generated by the supervised learning algorithm is feed as an input the perceptron network. This increases the level of the output provided to the patients or the doctors to a high precision. The flow diagram for the proposed working model is shown in figure 4

\section{RESULTS}

A care must be taken while implementing this system. The better use of this system when compared to CDSS is that it narrows down the list of the decisions to be made to a minimum number than the CDSS. This reduces the time a doctor must spend on the choices while using the system than the time it takes on CDSS. This increases the efficiency of the system with a better performance by providing better choices. Focusing only on functional decision making by the CDSS causes a deficient plan on how the doctors use the system in difficult situations. This system produces alerts only for the very less number of precise results that are given whereas the CDSS produce massive number of alerts as it gives a wide range of recommendations. During the 6 month observation period, 12,189 patients were hospitalized in the six internal medicine departments studied and received 136,459 medication prescriptions [5]. Among these prescriptions, $30.1 \%$ triggered one or more CDSS alerts each month per department [5]. When a system produces a high volume of results the doctors tend to ignore or pay less attention to the warnings causing potentially critical alerts to be missed. As the proposed method produce only important alerts after the whole classification is done, it will not lead to any annoyance and therefore helping doctors to look at every important alert that is produced. CDSS alerts cause interruption in the workflow, forcing the user to acknowledge the alert before proceeding with the prescribing, and are thus often considered a nuisance [6]. The neural networks system in CDSS fails to explain the reason for utilizing the data in a standard way for analyzing the patterns in the data and providing the decisions. The disadvantage of the CDSS is that the training process may take too much time based on the medical history and the symptoms of patients. In this system the data is combines based on recognizing the patterns and analyzing them alone which may not provide the proper decisions for the doctors to choose. The main barriers that are associated with the CDSS are feasibility, high number of alerts, high cost for integrating in work environment and the key fields of data that need to be addressed to avoid potentially adverse events from occurring which includes privacy, confidentiality, data accuracy and completeness, uniformity and alert desensitization. These are taken care of in the proposed methodology as the data is verified while implementing inference rules in both database and knowledge base resulting in producing only important decisions for the doctor.

\section{CONCLUSION}

The benefits and the efficiency of the clinical decision support systems on the doctor's performance and patient outcomes remain the subject of ongoing research. As the proposed method gives the precise number of decisions than the CDSS it is suggestible that the proposed method can be better implemented than the CDSS. Currently, each one of these studies must be carefully read and evaluated before implementing in an accurate way. Despite the advantages of using CDSS, there are also disadvantages such as data privacy. Safety measures are to be taken care of before implementing this system. There has always been errors that 
occur within the healthcare industry. Although many of the problems and barriers were overcome during this research, new challenges may arise during the implementation of the proposed method. The technical aspects of implementing both methods in this paper are challenging and many precautions should be taken as this system in the medical field is very sensitive and proper guidance along with the precision in the medical data plays a crucial role. The future work should be focusing on minimizing these errors as much as possible and thus providing a quality and an efficient patient healthcare systems. It is hoped that the future research works and projects shed more light on this system and implement the proposed method without any errors which will help doctors and patients by providing better healthcare support system.

\section{REFERENCES}

[1] Moja, L; Kwag, KH; Lytras, T; Bertizzolo, L; Brandt, L; Pecoraro, V; Rigon, G; Vaona, A; Ruggiero, F; Mangia, M; Iorio, A; Kunnamo, I; Bonovas, S (December 2014). "Effectiveness of computerized decision support systems linked to electronic health records: a systematic review and meta-analysis." American journal of public health 104 (12): e12-22.

[2] Wagholikar, K; Kathy L. MacLaughlin; Thomas M Kastner; Petra M Casey; Michael Henry; Robert A Greenes; Hongfang Liu; Rajeev Chaudhry (2013). "Formative evaluation of the accuracy of a clinical decision support system for cervical cancer screening". Journal of American Medical Informatics Association. pp. 747-759. Retrieved 6 March 2013.

[3] Rothman, Brian; Joan. C. Leonard; Michael. M. Vigoda (2012). "Future of electronic health records: implications for decision support". Mount Sinai Journal of Medicine 79 (6): 757-768.

[4] Venkata Karthik Gullapalli, Rahul Brungi, “A Novel Methodology to Implement Optimization Algorithms in Machine Learning", International Journal of Computer
Applications (0975 - 8887), Volume 112 - No 4, February 2015.

[5] Loya, S. R.; Kawamoto, K; Chatwin, C; Huser, V (2014). "Service oriented architecture for clinical decision support: A systematic review and future directions". Journal of Medical Systems.

[6] Yael Zenziper, Daniel Kurnik, Noa Markovits, Amitai Ziv, Ari Shamiss, Hillel Halkin and Ronen Loebstein, "Implementation of a Clinical Decision Support System for Computerized Drug Prescription Entries in a Large Tertiary Care Hospital”, IMAJ, volume 16, May 2014.

[7] Niazkhani Z, Pirnejad H, Berg M, Aarts J. The impact of computerized provider order entry systems on inpatient clinical workflow: a literature review. J Am Med .Inform Assoc 2009; 16: 539-49.

[8] Chaffee BW, Zimmerman CR. Developing and implementing clinical decision support for use in a computerized prescriber-order-entry system. Am J Health Syst Pharm. 2010 Mar 1; 67 (5):391-400.

[9] Bell LM, Grundmeier R, Localio R, Zorc J, Fiks AG, Zhang X, Stephens TB, Swietlik M, Guevara JP. Electronic health record-based decision support to improve asthma care: A cluster-randomized trial. Pediatrics. 2010 Apr; 125(4):e770-7.

[10] Venkata Karthik Gullapalli and Aishwarya Asesh, Data Trawling and Security Strategies, ISSN - 2278-8727, IOSR Journal of Computer Engineering, Volume 16, Issue 6, Ver. 1, Nov - Dec 2014.

[11] D Tadaki, "Advanced Surgical Imaging." Biomedical Science \& Engineering Conference, 2009, IEEE, pages 1 -1 .

[12] M. Frize, C. M. Ennett, M. Stevenson, H. Trigg. "Clinical decision support system for intensive care units: using artificial neural networks." Medical Engineering \& Physics, 2001, pages 217-225. 